\title{
CDISC SEND Challenge Agent Parameter Code Terminology
}

National Cancer Institute

\section{Source}

National Cancer Institute. CDISC SEND Challenge Agent Parameter Code Terminology. NCI Thesaurus. Code C158116.

Terminology associated with the challenge agent parameter code codelist of the Clinical Data Interchange Standards Consortium (CDISC) Standard for the Exchange of Nonclinical Data (SEND). 